\title{
LOS NUDOS GORGIANOS DE LAS DEMOCRACIAS LATINOAMERICANAS. ${ }^{1}$ \\ GORDIAN KNOTS OF LATIN \\ AMERICAN DEMOCRACIES.
}

Godofredo Vidal de la Rosa*

RESUMEN: Este trabajo explora la tensión entre los imperativos de Igualdad Política democrática y las tendencias seculares hacia la desigualdad social en América Latina. Básicamente el patrón que se examina es de alcance mundial y representa un reto muy serio para la teoría política y para la política real. En el trabajo se discuten los mecanismos qué convierten la desigualdad social en desigualdad política, y las acciones que contrarrestan la formación de políticas intratables (nudos gorgianos) en la polís democrática.

Palabras clave: equidad política; desigualdad social; desigualdad política; América latina; democracia.

\footnotetext{
${ }^{1}$ Texto presentado originalmente en el Congreso Joint Conference, Whatever Happened to North-South, São Paulo, Febrero 19, 2011.

"Doutor en Ciências Políticas y Sociales; Profesor Titular en el Departamento de Sociologia, División de Ciencias Sociales y Humanidades, Universidad Autónoma Metropolitana (UAM), Plantel Azcapotzalco, Ciudad de México, D. F., México; Email: gvdr@correo.azc.uam.mx
} 
ABSTRACT: This essay explores the tension between the Political Equality imperatives of Democratic politics and the secular trends toward social inequality in Latin America. Basically, the general patter examined is a Global Reach and represents a threat very serious for Political Theory as for real politics. In this work are discussed the mechanisms that transform social inequality in political inequality, and the actions that counteract the formation of political inectractabilities (Gordian Knots) in the democratic policy.

Keywords: political equality; social inequality; political inequality; Latin America; democracy.

\section{INTRODUCCIÓN}

Durante las últimas tres décadas, los regímenes democráticos han florecido en todo el mundo. De los 65 países caracterizados como democracias a finales del 1980, en la actualidad hay aproximadamente 122 países en los que la competencia electoral define la manera de acceder al poder político. Entre estos países existen enormes diferencias institucionales en el rendimiento y el régimen de la "calidad" democrática (Diamond, 2002; Munck y Verkiulen, 2002); pero todos estos casos se ajustan a la metáfora de la Tercera Ola, acuñado por el difunto Samuel Huntington (1991). Esta ola es uno de los fenómenos políticos más importantes de la era moderna. Sobre todo, representa una mejora significativa, aunque en muchos casos apenas sensida, en los derechos políticos para millones de habitantes en la Tierra. Sin embargo, el lado oscuro de este proceso consiste en la simultánea llega de la Era de Desigualdad Global. La Era de la desigualdad tiene al menos dos rasgos reconocibles. El primero es la marginación social, seguida de la política, que a veces está aún en ciernes, y la constitución de bunkers de poder político, sostenido en recursos financieros, administrativos y de inteligencia y poderío militar. El contraste entre ambos fenómenos es sorprendentemente creciendo y no sólo afecta a las nuevas democracias, sino es extensivo a las democracias más establecidas, como los Estados Unidos. Las desigualdades sociales y económicas están creciendo al 
mismo tiempo que millones de personas no sólo reclaman el derecho al voto, sino a ejercer sus derechos políticos plenamente. La tensión entre la Ola de la democratización y la Ola de desigualdad no podría ser más inestable y potencialmente explosiva. La igualdad política se erige como el imperativo político categórico, como decía el profesor Dahl (1983), al mismo tiempo que las desigualdades sociales y económicas impregnan la economía global. Estos fenómenos simultáneos están relacionados de muchas maneras. La desigualdad socioeconómica afecta de manera específica el ejercicio democrático y diseño institucional de las nuevas democracias, y la política democrática, paradójicamente, está detrás de la ola de la desigualdad social y económica.

Estos hechos son más dramática ya que en las ciencias políticas se considera como una cuestión fuera de debate, al menos normativamente, el que la democracia tiene que ver específicamente con las libertades civiles, pero no necesariamente con el desarrollo social o la justicia social. Excepto algunos notables filósofos, como Amatrya Sen, son pocos los que explícitamente vinculan la democracia con la justicia social. Por otra parte, las democracias tienen un record menos que satisfactorio que otros sistemas políticos modernos para producir el bienestar social o la igualdad social. ${ }^{2}$ Las obras seminales de Adam Przeworski y sus colegas (2000) apoyan la idea de que los estados democracias no son siempre el ganador en la carrera por el desarrollo y la distribución. La razón de este hecho está generalmente relacionada con la dinámica de los capitalistas. Por un lado, el desarrollo es un proceso largo, normalmente que involucra muchas generaciones, que implica una alta concentración de ingresos (Boix, 2003; Boix y Stokes, 2003; Acemoglu y Richardson, 2006). La idea se apoya en la llamada curva de Kuznets (Chong, 2001). Es hasta que el éxito del proceso de desarrollo, cuando la riqueza comienza a fluir a la virtud de abajo, y cuando se satisface la masa crítica de una clase media y una clase obrera dis-

\footnotetext{
${ }^{2}$ La exploración del hecho de que las democracias con frecuencia son incapaces de escapar de sus dilemas sociales, como la desigualdad social y la marginación se asocia a dos cosas. La primera es la dificultad de lograr un consenso duradero sobre este Problema social, excepto en condiciones extremadamente favorables (como la Inglaterra victoriana) y excepcionalmente provocada por retos existenciales, como la amenaza de guerra que pone en riesgo la dominación de las elites. En cambio las dictaduras pueden imponer objetivos sin consensos previos, movilizando recursos, para bien, las menos de veces, y para mal, la mayoría.
} 
puestas a contener sus demandas y a procesarlas por las vías de la competencia electoral. Y a sentirse menos inclinados a expresiones políticas dramáticas o salidas radicales de la situación actual. Ciero umbral de afluencia mejora las condiciones de supervivencia democrática, siempre y cuan do esta ofresca expectativas razonablemente congruentes con la experiencia, de que las oportunidades llegarán para todos. Esta imagen general de que el sistema es básicamente equitativo en la distribución transgneracional de oportunidades es enormentnt apreciado por la teoría política democrática. Y cuando este consenso en las oportunidades distribuidas equitativamente se altera, los conflictos distributivos no solo surgen desde los excluidos, sino sobre todo, de los privilegiados, que pueden preferir renunciar a la democracia que a sus ingresos y condiciones privilegiadas. Por supuesto esta tendencia es sólo una manera simple para describir un patrón general. Las anomalías ocurren. El ejemplo clásico es la India, donde la distribución del ingreso es muy mala, pero la democracia es una forma habitual, aunque sobresaltada, de hacer política. En los otros extremos son los países de América Latina, donde se implantó la democracia desde hace dos o tres décadas a pesar de las muy altas desigualdades.

Un caso particular es cuando la democracia llega antes de la cristalización típica de las etapas sociales y económicas asociadas a la modernización. La aparición de las democracias viables no se encuentra al final del proceso, pero en el medio del proceso de modernización. Para teoría de la modernización típicamente elaborada durante la Guerra fría - este caso representa una anomalía. De hecho, la democracia, en la que nuevos actores, como las clases medias y los obreros, con sus nuevas demandas de representación y rendición de cuentas y el aumento de las demandas sociales y económicas, se representa una sobrecarga a las capacidades del Estado y a la conducción del desarrollo político. Cuando es el caso, el autoritarismo fue promovido sin discreción en los centros de pensamiento dominantes en los países centrales y en las periferias. Durante décadas la democracia no resultó una mejor opción para las élites tecnocráticas y de la política de modernización. Cuando a finales de los 80 en el 
siglo XX este clima cambio y la modernización autoritaria empezó a ser cuestionada por sus ineficacias y incapacidades para gobernar, se abrió, oaera sorpresa de muchos, la idea de la democracia sin adjetivos, como una ideología improbable en el mundo. Pero la revolución democrática o tercera ola de democratización introdujo un nuevo descenso de categoría analítica al problema de la modernización y una nueva propio problema para la teoría política. Esta anomalía histórica particular, se ha convertido en el centro de un programa de investigación intensa y más intenso debate. De esa manera, la pregunta ahora es muy interesante. El observador optimista puede argumentar que en el largo camino la desigualdad no perturba la institucionalización de los regímenes democráticos. Sin embargo, debe ser útil recordar que las democracias europeas y Estados Unidos no fueron el resultado de un momento mágico de difusión de valores democráticos, sino un largo proceso de asimilación y gestión de conflictos (Mann, 1993). Sin embargo, creo que la verdadera cuestión no es si la democracia está en peligro, pero si la democracia puede romper los nudos políticos gordianos para abrir el camino a la equidad política y el bienestar social. En Europa occidental el "estado de bienestar" hizo el trabajo. Pero esos tiempos están muy lejos de nosotros. Es difícil imaginar la implementación Estado de Bienestar en América Latina de la misma manera que hace medio siglo Europa occidental. El desmantelamiento del Estado de Bienestar en todo el mundo es un presagio inequívoco. Lo peor de todo, las democracias pueden generar trampas "distributivos" regresivas. P La globalización abarca un menú de mecanismo para agravar las desigualdades (Milanovic, 2006). El segundo enfoque se deriva de estos conocimientos teóricos. El rendimiento y la "calidad" de los regímenes democráticos se ven afectados por los conflictos distributivos sociales y económicos de una manera decisiva. Los registros históricos nos enseñan sobre las formas en que las democracias viejas desarrollaron estrategias y políticas para promover la igualdad política, y también el bienestar social y económico, especialmente de los trabajadores. De alguna manera estas respuestas fueron inducidas por las demandas sociales, en las formas de los movimientos sociales y sindicales y la izquierda 
reformista. Aunque los expertos han encontrado que hay variantes de capitalismos responsivos al problema de la equidad social, y la construcción de los estados de bienestar difieren en los países nórdicos de Alemania, Francia y la Gran Bretaña, en todos los casos, fueron las respuestas de las élites políticas y económicas al desafío de la rebelión, la ilegitimidad y la ruptura social.

Este hecho es muy impactante, aunque introduce pesimismo sobre nuestra condición y capacidad de que los Estados democráticos sean capaces de editar una segunda era de reformas para enfrentar el nuevo Problema Social. El hecho real es que la tercera ola de democratización es también el gran tsunami de la desigualdad. Este trabajo es un intento de encontrar el nexo entre ambos fenómenos y centrarse en cuestión. ¿Existen mecanismos democráticos que trabajan en contra de la sociedad más igualitaria?

\section{La era de la desigualdad}

El tsunami real de desigualdad social y económica no tiene precedentes en la historia. Las diferencias entre los ingresos entre los países están creciendo rápidamente y profundamente. Incluyendo los EE.UU. y Europa Occidental se ven afectados por esta regresión de $\mathrm{v}$ los ingresos de las familias.

Un informe de los equipos de American Political Science Association (APSA) (2008) advierte sobre este proceso en los Estados Unidos que se agrava especialmente desde los años 80 del siglo $\mathrm{XX}$ (también Trent-Echeverri, 2009). Los países de América Latina son particularmente vulnerables, y especialmente durante la última década del siglo pasado, la distribución del ingreso ha empeorado, arrojando a millones de las personas a la marginalidad y la pobreza. En el caso de América Latina este hecho es más preocupante, ya que se une a la herencia histórica de la mala distribución y la exclusión social. La fragilidad latinoamericana tiene un rasgo interesante. Ocurre por ciclos decenales. Los años 90 se calificaron como "décadas perdidas" por los efectos de los ajustes fiscales y los cambios en las políticas económicas en el contexto de la hegemonía neoliberal. Pero la pri- 
mera década de este siglo se presumieron avances importantes en los programas contra la marginación extrema y se impulsaron programas de bienestar social extensos. Pero en la segunda década del siglo, las advertencias de regresiones y un repunto del problema de la exclusión y marginación aparece como una tormenta amenazante.

Este documento se centra en la región de América Latina y explora la forma en que el creciente empobrecimiento y la desigualdad afecta la política de la democracia. Por lo menos hay dos puntos de vista sobre el problema. El primero insiste en que la democracia no está en peligro por la brecha de la desigualdad. Las instituciones democráticas son viables y están en un proceso de consolidación. Los defensores de esta tesis reeditan la tesis de Huntington, impulsada hace cuatro décadas (1967), que sugiere que la pobreza no es la causa de las revoluciones o revueltas, como si lo es la percepción de la exclusión social y política (Kauffman, 2009). Pero siendo optimista, se puede argumentar que la democracia tal vez no puede promover la justicia social o la mejora social, pero la inclusión política (Bermeo 2009). El avance y credibilidad da igualdad política, puede contener, al menos temporalmente las demandas por soluciones a las desigualdades sociales y económicas y ambos procesos, el político y el social, separados parcialmente por una hábil conducción política. Esta tesis está en tensión con la teoría democrática moderna. Por ejemplo, Robert Dahl (1971) pone gran atención a los complejos vínculos entre la igualdad política y conflictos distributivos sociales y económicos y el Premio Nobel de Economía Amartya Sen siguió la misma forma de pensar cuando identificó la democracia con los avances sustanciales en las libertades políticas y la ampliación de las capacidades humanas. 
Gráfico I - Inequidad 1950 - 2000

\section{Inequidad, 1950-2000: La madre de todas las disputas}

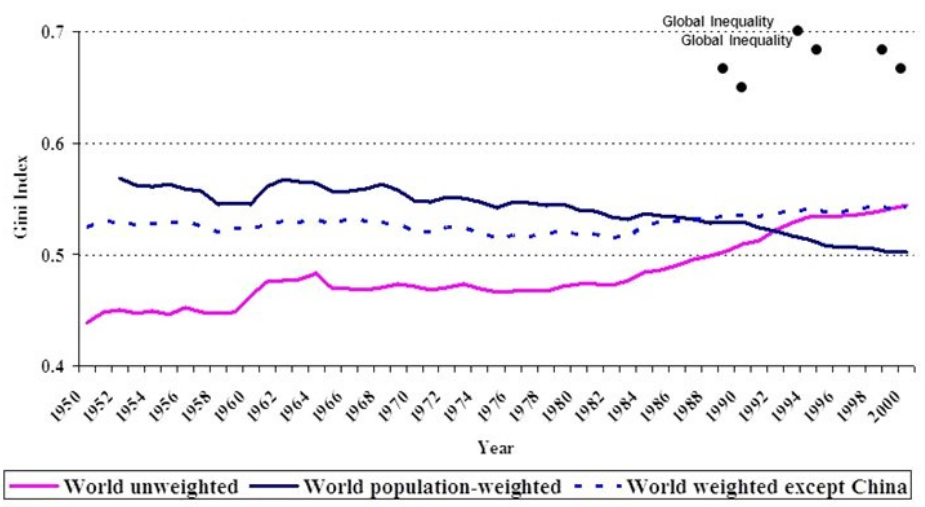

Fuente: Milanovic, 2005.

A pesar de las mejoras parciales aunque considerables en el desarrollo humano relativo, en América Latina, los conflictos distributivos parecen constituir un foco importante de la política democrática. Contrariamente a los analistas que insisten en la idea de que los conflictos distributivos no son un problema importante para la consolidación democrática, el "giro a la izquierda" en América del Sur da un fuerte apoyo a la Dahl y Sen. En este artículo voy a preceder primero, analizando el "giro de la izquierda" y en segundo lugar, examinare el desempeño de la política de los gobiernos "izquierdistas" de la región. Como hay considerables diferencias en las experiencias nacionales, explorar sus características y posibilidades es un tema importante para los científicos políticos. 
Gráfico II - Índice de desarrollo humano de países seleccionados de América Latina

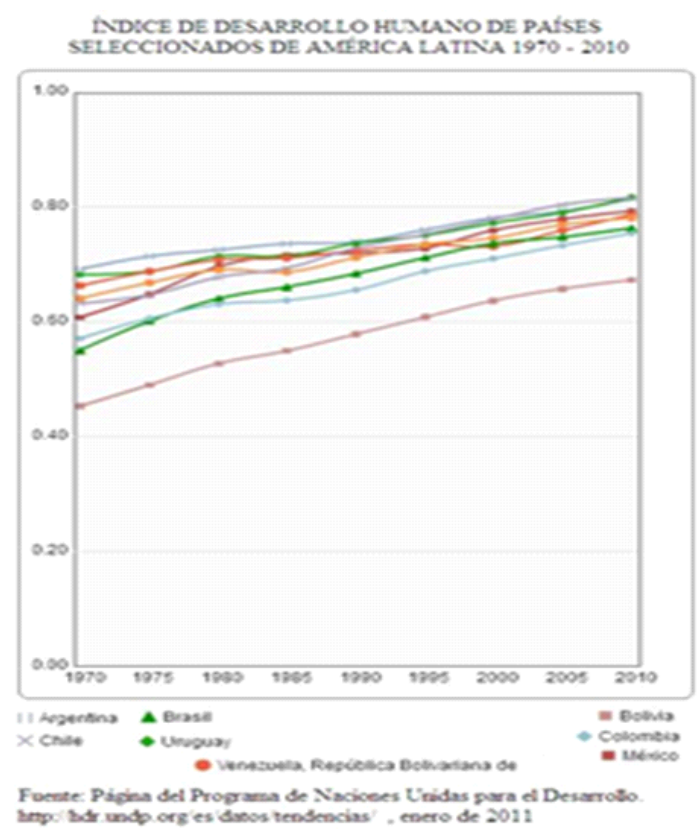

La forma de proceder aquí es el siguiente. En primer lugar, le doy un panorama descriptivo de las tendencias de la onda de Transición en América Latina, nos dividimos en dos mitades. La etapa neoliberal y el giro izquierdista. Cada fase tiene tres aspectos: las reformas económicas, reformas políticas y de inclusión o de participación política. El éxito de los países de América Latina es desigual, y quiero encontrar algunos rasgos suficientes visibles a generalizaciones creíbles hechas. En segundo lugar, hago algunas indicaciones sobre la pertinencia de nuevos avances en la investigación teórica y empírica.

\section{El giro neoliberal}

Durante los años de reciente formación democrática, la mayoría de los países de la región experimentaron intensos cambios económicos. Muchas de las reformas llamadas "neoliberales", tuvie- 
ron "efectos profundos". Para algunos analistas, los efectos limitados sobre el crecimiento fueron causados por las reformas neoliberales aplicadas de manera incompleta; el argumento implica que cuando se aplicaron las reformas de manera sistemática, como en Chile, la economía estaba en una buena forma, y cuando se aplicaban sin decisión o con incompetencia, los resultados eran menos que destrozos. La decisión y liderazgo de una coalición política comprometida con la agenda neoliberal sería, en este razonamiento, la clave para el éxito económico. .La conclusión es que las reformas no neoliberales sino la insuficiente aplicación era la principal causa del mal funcionamiento del entorno macroeconómico. Sin embargo, es más creíble la idea de que la agenda neoliberal ideal era una medicina demasiado amarga para ser tragada sin grandes alteraciones en el orden político, social, económico y aun en las capacidades estatales. De hecho, el registro de los, logros de la agenda neoliberal ha sido mediocre, con excepción de países relativamente pequeños, como Chile. En México y Brasil, las agendas neoliberales implantadas a raíz de los primeros años de las transiciones, y en medio de gravísimas crisis económicas y consecuencias sociales penosas, han sido menos que satisfactorios (Cohen y Centeno, 2006). En el caso de América Latina la agenda neoliberal tiene resultados mediocres similares (CEPAL). Pero este ensayo no puede acercarse a los resultados generales de las reformas neoliberales ni el llamado proceso de globalización en profundidad. Para mi propósito es suficiente para notar los efectos "colaterales daños" de la globalización sobre la desigualdad y la exclusión social.

Durante la última década del siglo pasado, la distribución del ingreso se deterioró o se mantuvo estable en los altos niveles de desigualdad. La distribución del ingreso no es una medida directa de la desigualdad política, sino un indicador de por poder. Pero es razonable suponer que si la distribución del ingreso mantiene una alta desigualdad durante largos períodos de tiempo y / o asociada a un conjunto específico de las políticas, refleja un hecho político. Suponiendo que criterios como la principal hipótesis conducirán a la empresa productiva de entender el mecanismo que provoca la desigualdad -y exclusiónsocio-política y los efectos de la exclusión en sí. 
Gráfico III - Coeficiente de Gini por grandes regiones geográficas - Año 2004

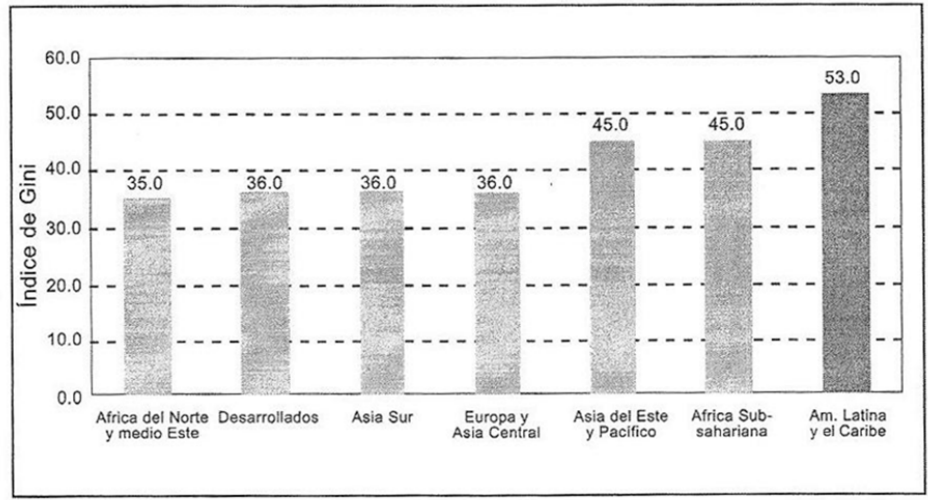

Fuente: Ferreira y Ravallion (2008).

A finales de los tres decenios, las desigualdades de ingresos crecieron mucho. Este deterioro se debe resumirse a la desigualdad social y económica extrema anterior de la región. Las democracias latinoamericanas se sustentan en desigualdades gigantescas. Por ejemplo las democracias de Europa Occidental tienen un índice de GINI mediana de la distribución del ingreso mucho menos atenuada.

Gráfico IV - Desigualdad en América Latina por región Coeficiente de Gini Distribución del ingreso per cápita (no ponderado)

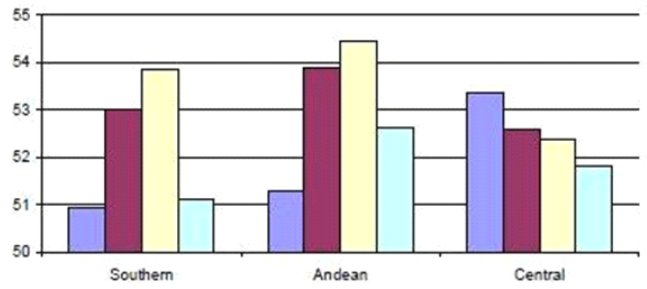

口Early 1990s QLate 1990s DEarly 2000s Q Mid 2000s

Fuente: Gasparini et al., 2009 
La desigualdad no es sólo un fenómeno político. La privación de recursos como la educación, la alimentación, los ingresos por trabajo, los servicios sociales como la salud y la vivienda, disminuyen la capacidad de ejercer los derechos ciudadanos. La desigualdad social afecta la distribución de los costos para participar, elevando los de los grupos excluidos. Si los costos de participar efectivamente se elevan, también los riesgos de coacción, represión y maltrato cívico. La desigualdad tiene que ver con el malestar social, la delincuencia y la violencia, la marginalidad y la exclusión. La desigualdad no sólo es un daño colateral temporal de modernización, pero un cáncer dramática que crece dentro. Pueden surgir mecanismos compensatorios, como acciones colectivas, en forma de coaliciones políticas nuevas, organizaciones sindicales y sociales que disminuyan la brecha de la desigualdad política, pero solo si logran afectar la distribución de oportunidades a los bienes públicos. En tanto, la ventaja es para los grupos favorecidos y las coaliciones políticas que los representan. Debido a los problemas de desigualdad están en todas partes, los gobiernos pueden tener la responsabilidad primordial de ofrecer políticas y respuestas para mejorar y eventualmente resolver los aspectos más inmediatos de la pobreza extrema y la exclusión. Pero para eso, nuevamente, deben construir coaliciones suficientemente estables y alicientes suficientemente convincentes, para articular políticas gubernamentales reformistas. $\mathrm{O}$ bien, las coaliciones conservadoras del status quo pueden concluir que la desigualdad y el "problema social" es suficientemente real y candente, para ofrecer paliativos a la protesta y la desgobernabilidad. Este hecho puede explicar parcialmente las oleadas de un nuevo enfoque del desarrollo a principios de este siglo en América del Sur. (OEA-PNUD, 2010). 
Tabula I -América Latina: distribución del ingreso por hogares, 1990-2002

\begin{tabular}{|c|c|c|c|c|c|c|c|c|}
\hline \multirow[t]{3}{*}{ País } & \multirow[t]{3}{*}{ Año } & \multirow{2}{*}{\multicolumn{2}{|c|}{$\begin{array}{c}\text { Participación en el } \\
\text { ingreso total del }\end{array}$}} & \multirow{3}{*}{$\begin{array}{l}\text { Cociente } \\
10 \% / 40 \%\end{array}$} & \multicolumn{4}{|c|}{ Índice de concentración } \\
\hline & & & & & \multirow[t]{2}{*}{ Gini } & \multirow{2}{*}{$\begin{array}{c}\text { Varianza } \\
\text { logarítmica }\end{array}$} & \multirow[t]{2}{*}{ Theil } & \multirow[t]{2}{*}{ Atkinson } \\
\hline & & $40 \%$ más pobre & $10 \%$ más rico & & & & & \\
\hline \multirow[t]{4}{*}{ Argentina $^{\mathrm{b}}$} & 1990 & 14,9 & 34,8 & 2,3 & 0,501 & 0,982 & 0,555 & 0,570 \\
\hline & 1997 & 14,9 & 35,8 & 2,4 & 0,530 & 1,143 & 0,601 & 0,607 \\
\hline & 1999 & 15,4 & 37,0 & 2,4 & 0,542 & 1,183 & 0,681 & 0,623 \\
\hline & 2002 & 13,4 & 42,1 & 3,1 & 0,590 & 1,603 & 0,742 & 0,702 \\
\hline \multirow[t]{4}{*}{ Bolivia } & $1989^{c}$ & 12,1 & 27,9 & 2,3 & 0,538 & 1,528 & 0,574 & 0,771 \\
\hline & 1997 & 9,4 & 27,9 & 3,0 & 0,595 & 2,024 & 0,728 & 0,795 \\
\hline & 1999 & 9,2 & 29,6 & 3,2 & 0,586 & 2,548 & 0,658 & 0,867 \\
\hline & 2002 & 9,5 & 28,3 & 3,0 & 0,614 & 2,510 & 0,776 & 0,865 \\
\hline \multirow[t]{4}{*}{ Brasil } & 1990 & 9,5 & 43,9 & 4,6 & 0,627 & 1,938 & 0,816 & 0,790 \\
\hline & 1996 & 9,9 & 46,0 & 4,6 & 0,638 & 1,962 & 0,871 & 0,762 \\
\hline & 1999 & 10,1 & 47,1 & 4,7 & 0,640 & 1,913 & 0,914 & 0,754 \\
\hline & 2001 & 10,2 & 46,8 & 4,6 & 0,639 & 1,925 & 0,914 & 0,760 \\
\hline \multirow[t]{3}{*}{ Chile } & 1990 & 13,2 & 40,7 & 3,1 & 0,554 & 1,258 & 0,644 & 0,671 \\
\hline & 1996 & 13,1 & 40,2 & 3,1 & 0,553 & 1,261 & 0,630 & 0,667 \\
\hline & 2000 & 13,8 & 40,3 & 2,9 & 0,559 & 1,278 & 0,666 & 0,658 \\
\hline \multirow[t]{4}{*}{ Colombia } & 1994 & 10,0 & 41,8 & 4,2 & 0,601 & 2,042 & 0,794 & 0,817 \\
\hline & 1997 & 12,5 & 40,1 & 3,2 & 0,569 & 1,399 & 0,857 & 0,822 \\
\hline & 1999 & 12,3 & 40,1 & 3,3 & 0,572 & 1,456 & 0,734 & 0,945 \\
\hline & $2002^{\mathrm{d}}$ & 11,9 & 39,1 & 3,3 & 0,575 & 1,413 & 0,714 & 0,701 \\
\hline \multirow[t]{4}{*}{ Costa Rica } & 1990 & 16,7 & 25,6 & 1,5 & 0,438 & 0,833 & 0,328 & 0,539 \\
\hline & 1997 & 16,5 & 27,3 & 1,7 & 0,450 & 0,860 & 0,356 & 0,535 \\
\hline & 1999 & 15,3 & 29,4 & 19 & 0,473 & 0,974 & 0,395 & 0,573 \\
\hline & 2002 & 14,5 & 30,2 & 2,1 & 0,488 & 1,080 & 0,440 & 0,646 \\
\hline \multirow[t]{4}{*}{ Ecuador $^{d}$} & 1990 & 16,7 & 25,6 & 1,5 & 0,461 & 0,823 & 0,403 & 0,591 \\
\hline & 1997 & 16,5 & 27,3 & 1,7 & 0,469 & 0,832 & 0,409 & 0.510 \\
\hline & 1999 & 15,3 & 29,4 & 1,9 & 0,521 & 1,075 & 0,567 & 0,597 \\
\hline & 2002 & 14,5 & 30,2 & 2,1 & 0,513 & 1,031 & 0,563 & 0,593 \\
\hline \multirow[t]{4}{*}{ México } & 1989 & 15,8 & 36,6 & 2,3 & 0,536 & 1,096 & 0,680 & 0,598 \\
\hline & 1998 & 15,1 & 36,7 & 2,4 & 0,539 & 1,142 & 0,634 & 0,599 \\
\hline & 2000 & 14,6 & 36,4 & 2,5 & 0,542 & 1,221 & 0,603 & 0,621 \\
\hline & 2002 & 15,7 & 33,2 & 2,1 & 0,514 & 1,045 & 0,521 & 0,571 \\
\hline \multirow[t]{3}{*}{ Perú } & 1997 & 13,4 & 33,3 & 2,5 & 0,532 & 1,348 & 0,567 & 0,663 \\
\hline & 1999 & 13,4 & 36,5 & 2,7 & 0,545 & 1,358 & 0,599 & 0,673 \\
\hline & 2001 & 13,4 & 33,5 & 2,5 & 0,525 & 1,219 & 0,556 & 0,636 \\
\hline \multicolumn{9}{|l|}{ República } \\
\hline \multirow[t]{2}{*}{ Dominicana } & 2000 & 11,4 & 38,8 & 3,4 & 0,554 & 1,250 & 0,583 & 0,635 \\
\hline & 2002 & 12,0 & 38,3 & 3,2 & 0,544 & 1,216 & 0,570 & 0,637 \\
\hline Uruguay $^{\mathrm{d}}$ & 1990 & 20,1 & 31,2 & 1,6 & 0,492 & 0,812 & 0,699 & 0,519 \\
\hline & 1997 & 22,0 & 25,8 & 1,2 & 0,430 & 0,730 & 0,336 & 0,475 \\
\hline & 1999 & 21,6 & 27,0 & 1,3 & 0,440 & 0,764 & 0,354 & 0,483 \\
\hline & 2002 & 21,6 & 27,3 & 1,3 & 0,455 & 0,802 & 0,385 & 0,661 \\
\hline $\begin{array}{l}\text { Venezuela } \\
\text { (República }\end{array}$ & & & & & & & & \\
\hline Bolivariana de) & 1990 & 16,7 & 28,7 & 1,7 & 0,471 & 0,930 & 0,416 & 0,545 \\
\hline & 1997 & 14,7 & 32,8 & 2,2 & 0,507 & 1,223 & 0,508 & 0,985 \\
\hline & 1999 & 14,6 & 31,4 & 2,2 & 0,498 & 1,134 & 0,464 & 0,664 \\
\hline & 2002 & 14,3 & 31,3 & 2,2 & 0,500 & 1,122 & 0,456 & 0,866 \\
\hline
\end{tabular}

\section{Transición democrática}

Pero antes de continuar con este argumento es conveniente volver a la tesis de que la desigualdad amenaza la estabilidad democrática. Hay algunos estudiosos que sostienen que la creciente desigualdad no representa un peligro real para la democracia (Kauffman, 2009) y sostienen que las políticas públicas que tratan de reducir la pobreza demasiado rápido puede ser capturado por las fuerzas radicales y populismo (populismo especialmente la izquierda), y pide la prudencia 
(Bermeo, 2009). Sin embargo, la mayoría de los estudiosos están convencidos de que el aumento de la marginación y el empobrecimiento son amenazas sociales y políticas reales a la existencia social y política. Este argumento no es nuevo impregna las reformas liberales de Europa occidental a finales del siglo XIX, y los EE.UU. durante el New Deal (aunque también del surgimiento del régimen nazi en Alemania a mediados del siglo XX). Las reformas políticas y las políticas económicas distributivas son la alternativa históricamente preferida hasta ahora frente a una pauperización masiva y la marginación social. ${ }^{3}$ Además, la mayoría de análisis subestiman que la democracia no es muy eficaz como régimen distributivo. La razón fundamental de esta visión pesimista se deriva principalmente de la teoría de la elección social y el sesgo conservador. Pero si la democracia es una menos que el régimen regular para promover políticas distributivas, el desafío de América Latina es considerable.

Los tipos de regímenes que surgieron en el proceso de transición estaban lejos de cualquier ideal o cualquier norma de la democracia moderna avanzada. En estos tiempos, las palabras comunes son democracias parciales, democracias híbridas; democracias delegativas fue un término acuñado por el politólogo argentino Guillermo O’Donnell para referirse a un caso especial se presidencialismo gobernó sin restricciones característicos de los regímenes más avances. Rendición de cuentas, la división de poderes y la revisión judicial eran débiles instituciones de las nuevas democracias. En forma más general, las deficiencias y limitaciones democráticas fueron explicadas por tres tipos de causas y estos defectos o deficiencias son decisivos para el diferente estado de las capacidades de élite para la construcción de estrategias de inclusión (o no). Los primeros fueron el neoliberalismo en sí. Aunque esta explicación puede ser simplista, tiene cierta lógica. Para hablar de la forma estructural para integrar la economía nacional en la economía mundial es más preciso. La tesis original lo afirmó Raúl Presbisch y más tarde por Fernando Fanjzylver y Fernando Enrique Cardoso. La teoría ex-

${ }^{3}$ Recientemente ha surgido un interés renovado por los mecanismos capitalistas de distribución de los excedentes, y los mecanismos de apropiación de la riqueza en los cuales el sistema capitalista inherentemente induce desigualdad, y si no hay intervención social y política regulatoria, no hay limite a la acumulación excluyente (Piketty, 2014; un comentario interesante es el de Hopkins, 2014) 
presa que los vínculos estructurales de las economías internas con más economías se vieron reflejados en la formación y el poder de distribución de las élites. Por supuesto, la estrategia nacional más desarrollista debía reflejarse en una más compleja burguesía, menos oligárquica, y una orientación más nacionalista. Por extensión, esta tesis arregla muy bien con un modelo desarrollista de la democracia. En otra dirección, los vínculos externos (de dependencia), basado en las exportaciones primarias, generan arquitectura poder oligárquico menos apto para democratizar. Por cierto, esta teoría fue resucitada tratando de explicar las perspectivas de la democracia derivadas de la globalización (Solimano, 2005). En el 90 'las expectativas eran muy altas y una simbiosis se ha creado entre la expectativa de apertura democrática y el grado de apertura externa y la liberalización. A la vuelta del siglo, la tesis muestra sus fracasos, pero contribuyó a revitalizar el interés por las explicaciones de economía política sobre los tipos de imbricaciones entre los mercados y las agencias estatales de regulación y de desarrollo. En términos de la teoría democrática el principal problema es si las instituciones estatales son capaces de regular las actividades de mercado, y para evadir el proceso de captura y la influencia política de las elites económicas en el proceso político. Tentativamente, un compromiso de no-non-neoliberalismo no ortodoxa con el proceso de globalización es una indicación de la conciencia élites y la apertura a los cambios económicos y políticos.

El otro conjunto de fenómenos fue el sistema de partidos. Esta variable es interesante porque los partidos son el canal a la representación y la participación política. Sin una competencia electoral dinámico y enérgico que no hay posibilidad de representación electoral. En otro modo, se detectó una crisis de la representación en forma de sistemas de partidos restringida. Las trampas democráticas que bloquean las demandas distributivas están bien estudiados. En primer lugar, es el camino observado para las personas más pobres para evitar la participación en la política ni en los procesos electorales.

Pero los sistemas de voto obligatorio son un remedio contra esta pasividad si hay las expectativas de que el acto de votación tienen significado y las consecuencias para las personas comunes. Si no 
hay un sistema de partidos, que ofrece una plataforma política y el funcionamiento político percibido como eficaz, las personas no van a las urnas. Sistemas de voto obligatorio no resuelven la eficiencia de votación sin un sistema de partidos que los canales de las preferencias de una manera convincente o creíble. La aparición de estas nuevas coaliciones de izquierda impulsó una nueva ola de legitimidad al proceso democrático y la participación política. Cuando la formación y actividad estas nuevas coaliciones están bloqueadas (el costo de la acción colectiva es alta), podemos observar no sólo mayor abstención, pero creciente insatisfacción con la democracia (véase el informe de 2010 de la Latino Barómetro).

En América Latina el estándar preferido es el voto obligatorio. En teoría, el voto obligatorio es una buena opción si el objetivo político es obtener una alta participación. Añadido a ese factor, hay cierta evidencia de que, al menos potencialmente, dice que si no hay un sistema de partidos sana y competitiva, el voto obligatorio se incrementará la probabilidad de que las clases bajas para participar en la competencia política electoral. Sin embargo, a pesar de los esquemas de voto obligatorio se aplican en la mayoría de los países de América Latina, los niveles de abstención son más grandes que en el resto del mundo. El voto obligatorio puede explicar los niveles de participación relativamente más altos en Brasil. ${ }^{4}$

Gráfico V - El sufragio: población de votantes por regiones

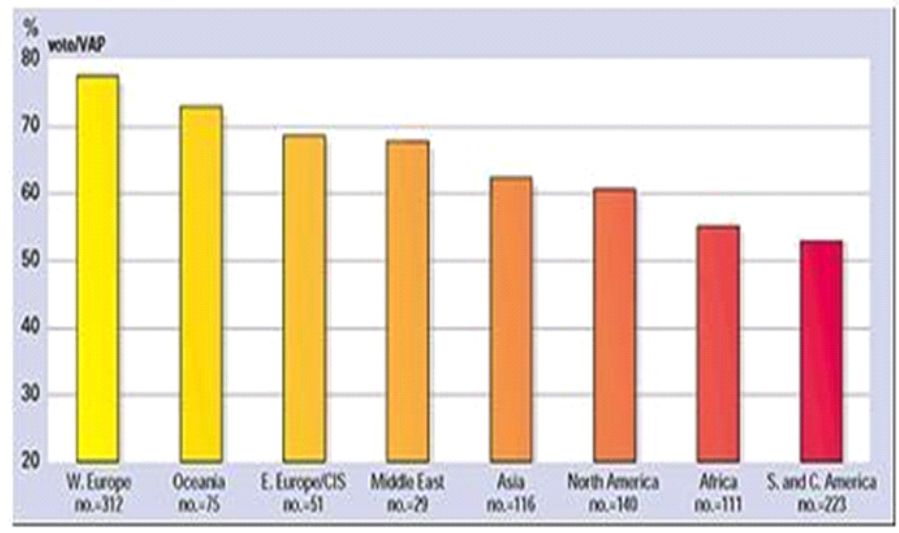

Fuente: IDEA (www idea.int) 
Dado que los países de América Latina tienen una media más baja entre los continentes, los comportamientos electorales son muy diferenciados. Países del Continente Sur han alcanzado niveles de participación electoral similar a Europa, pero México y Colombia mantienen las tasas más bajas de participación electoral. La explicación de la diferencia tesis ha sido proponed en la institución del sistema obligatorio de votación. La obligatoriedad del voto es cuestionada en círculos liberales por que van en contra de la libertad individual, aunque se puede argumentar que al contrario, son una de las responsabilidades de la ciudadanía con el Bien Común. Los sistemas obligatorios potencialmente aumentan las posibilidades de que la ciudadanía marginada. En vez de anular su voto el ciudadano debe elegir entre ofertas partidistas. Este hecho no aumenta el riesgo de actividades clientelistas de los partidos, Asi que el voto obligatorio parece inútil sin una competencia partidista real e imparcial. Sin embargo, su es razonable asumir que los sistemas obligatorios pueden ser inductores útil de voto cuando hay una fuerte resistencia de los votantes a votar. ${ }^{5}$

\footnotetext{
${ }^{5}$ Todos los países de Sur América (con la excepción de Colombia, Venezuela (donde fue abolido en 1994) y México, tienen sistemas de voto "obligatorio", que legados de experiencias democráticas previas (Argentina, 1912; Bolivia, 1929; Brasil, 1932; Chile, 1919. Un sistema extraño donde votan obligatoriamente los inscritos en el padrón, pero la inscripción es voluntaria); Perú, 1931 (alfabetos) y 1980 universal, Uruguay. 1916; Venezuela, 1947 (Fernández Baeza, 1988). El voto de las mujeres, a nivel nacional, fue reconocido, en Uruguay, en 1927. En Brasil en 1932, Argentina, en 1947, Venezuela, 1947, en Chile en 1949, en México, en 1953. Colombia, 1957. (Bethell, 1997, p.41)
} 


\section{El voto en américa latina}

\begin{tabular}{|c|c|c|c|}
\hline País & Tipo de elección & $\begin{array}{c}\text { Votos } \\
\text { efectivos }\end{array}$ & Registro \\
\hline \multirow{2}{*}{ Argentina } & Parlamentaria & & 73.13 \\
\hline & Presidencial & $71.81 \%(2007)$ & $27,090,236(2007)$ \\
\hline \multirow{2}{*}{ Brasil } & Parlamentaria & $83.27 \%(2006)$ & $125,827,119(2006)$ \\
\hline & Presidencial & $83.25 \%(2006)$ & $125,913,479(2006)$ \\
\hline \multirow{2}{*}{ Chile } & Parlamentaria & $87.67 \%(2005)$ & $8,220,897(2005)$ \\
\hline & Presidencial & $84.43 \%(2006)$ & $8,220,897(2006)$ \\
\hline \multirow{2}{*}{ Colombia } & Parlamentaria & $40.49 \%(2006)$ & $26,595,171$ (2006) \\
\hline & Presidencial & $45.11 \%(2006)$ & $26,731,700(2006)$ \\
\hline \multirow{2}{*}{ Costa Rica } & Parlamentaria & $65.13 \%(2006)$ & $2,156,572(2006)$ \\
\hline & Presidencial & $65.21 \%(2006)$ & $2,550,613(2006)$ \\
\hline \multirow{2}{*}{ Cuba } & Parlamentaria & - & - \\
\hline & Presidencial & - & - \\
\hline \multirow{2}{*}{$\begin{array}{l}\text { República } \\
\text { Dominicana }\end{array}$} & Parlamentaria & $56.46 \%(2006)$ & $5,492,880(2006)$ \\
\hline & Presidencial & $71.36 \%(2008)$ & $5,764,387(2008)$ \\
\hline \multirow{2}{*}{ Ecuador } & Parliamentaria & $63.50 \%(2006)$ & $9,165,125$ (2006) \\
\hline & Presidencial & $76.01 \%(2006)$ & $9,165,125(2006)$ \\
\hline \multirow{2}{*}{ El Salvador } & Parlamentaria & $53.58 \%$ (2009) & $4,226,479$ (2009) \\
\hline & Presidencial & $61.91 \%$ (2009) & $4,294,849$ (2009) \\
\hline \multirow{2}{*}{ Guatemala } & Parlamentaria & $60.46 \%$ (2007) & $5,990,029(2007)$ \\
\hline & Presidencial & $48.15 \%$ (2007) & $5,990,029(2007)$ \\
\hline \multirow{2}{*}{ Honduras } & Parlamentaria & $45.97 \%(2005)$ & $3,988,605(2005)$ \\
\hline & Presidencial & $55.08 \%(2005)$ & $3,976,550(2005)$ \\
\hline \multirow{2}{*}{ México } & Parlamentaria & $58.90 \%(2006)$ & $71,351,585$ (2006) \\
\hline & Presidencial & $58.55 \%(2006)$ & $71,374,373(2006)$ \\
\hline \multirow{2}{*}{ Nicaragua } & Parlamentaria & $66.73 \%(2006)$ & $3,665,141(2006)$ \\
\hline & Presidencial & $61.23 \%(2006)$ & $3,665,141(2006)$ \\
\hline \multirow[b]{2}{*}{ Panáma } & Parlamentaria & $76.27 \%(2004)$ & 1,999,553 (2004) \\
\hline & Presidencial & $76.90 \%(2004)$ & $1,999,628(2004)$ \\
\hline \multirow{2}{*}{ Per } & Parliamentaria & $88.66 \%$ (2006) & $16,494,906(2006)$ \\
\hline & Presidencial & $87.71 \%(2006)$ & $16,494,906(2006)$ \\
\hline \multirow{2}{*}{ Puerto Rico } & Parliamentaria & - & - \\
\hline & Presidencial & - & - \\
\hline \multirow{2}{*}{ Uruguay } & Parliamentaria & $89.62 \%(2004)$ & $2,487,816(2004)$ \\
\hline & Presidencial & $88.29 \%(2004)$ & $2,487,816(2004)$ \\
\hline
\end{tabular}

Fuente: IDEA 
Estas variables o dimensiones políticas, describen el significado de la crisis política. Es decir, la desigualdad y la creciente desigualdad en una arena democrática, significa un potencial de conflicto social y político. Existen diferentes estrategias para hacer frente a ese desafío. Elite en el poder puede elegir a relegar la amenaza, o para responder a ella de la manera paternalista, populista, o tratando de mejorar las instituciones democráticas políticas que pueden deteriorar o bloquear los desafíos políticos a adoptar canales democráticos. El primero es el llamado "giro a la izquierda" y el segundo, el éxito de los gobiernos "izquierdistas" en la promoción de políticas de lucha contra la pobreza.

Básicamente tenemos un gradiente que contiene un estereotipo de las salidas políticas a la crisis percibida derivada del aumento de la marginación. La tesis principal es que las reformas sociales y políticas son el mejor esfuerzo para mantener el status quo, sino que hay muy diferentes respuestas a la crisis desigualdad en las elites latinoamericanas. Las diferentes respuestas deben ser explicadas en términos de dimensiones estructurales, institucionales e históricas.

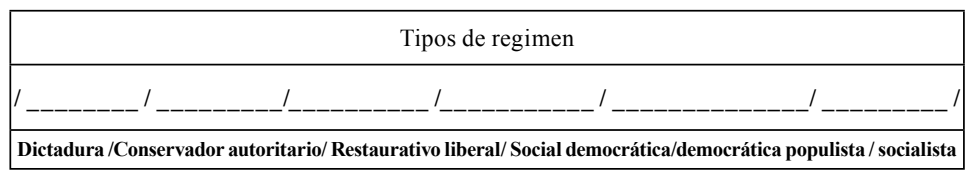

Cuando hay un sistema de partidos estable o consolidada la salida se ha traducido en una competición sistema de partidos plural, como en Brasil y Chile; cuando el acceso de nuevas coaliciones políticas ha sido bloqueado por las élites statu quo, las respuestas populistas son comunes. La otra salida es cuando los partidos de izquierda son débiles o fracturado o el costo de la acción colectiva es mayor debido a la compresión o la violencia o fraude político, el status quo se mantiene, pero a costa de un deterioro de la legitimidad política y la estabilidad del régimen (México, Colombia). La existencia de un sistema de partidos de competencia sólida y plural y estable es la variable clave en el éxito continuo de la participación política. 


\section{El regreso de la izquierda}

Con una alta votación, la percepción de la crisis es el factor principal del éxito electoral de partidos de izquierda y coaliciones. "Votación económica" se ha detectado como el principal factor en el impulso de la votación de izquierda (Murillo, et. Al., 2010). El estancamiento económico como resultado de las políticas macroeconómicas ortodoxas no es suficiente para inducir la votación económica. Para traducir el descontento político y social en los resultados políticos es necesaria la existencia de instituciones políticas y la confianza social del bajo riesgo de la represión. Debido a que en general se acepta que un registro de larga data de respeto de los derechos humanos debe ser la primera condición para alta participación política y electoral voluntaria. Los principales canales institucionales de participación política son un sistema de partidos y el sistema electoral.

La salud de ambos es decisiva para darle un poco de sostener la tesis voto económico. Sin estos canales institucionales, la votación económica se disipa en el engaño político a pesar de las reglas como el sistema de voto obligatorio. Pero en condiciones de juego limpio, en un sistema competitivo, abierto, las elecciones se están convirtiendo en una rendición de cuentas extensa mecanismo muy eficaz. 
Gráfico VI - Cambio porcentual del coeficiente de Gini por tipo de gobierno entre 2003 y 2006

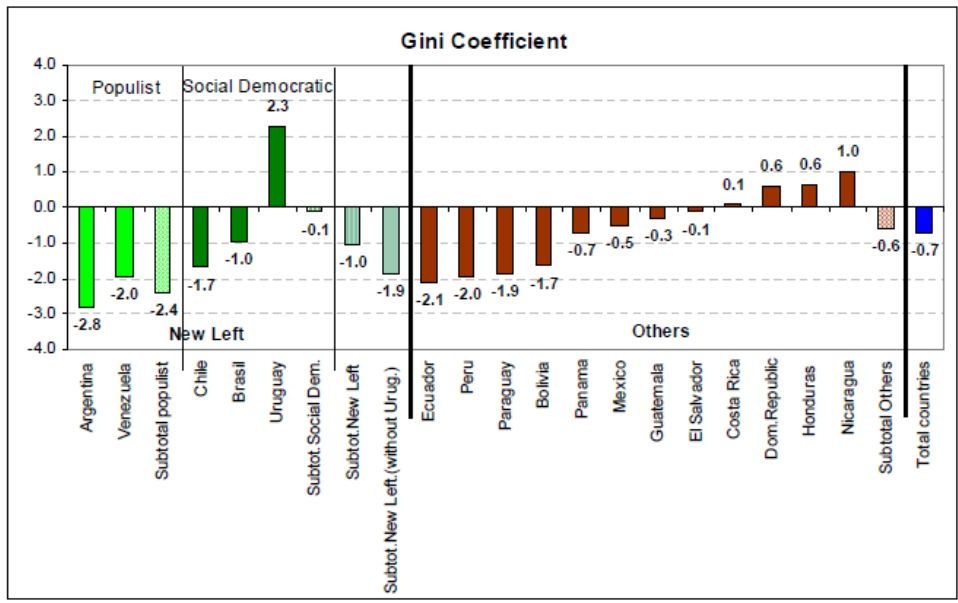

Gráfico: CEPAL, Nora Lusting.

El éxito de los gobiernos "izquierdistas" ha sido cuestionado en el trabajo de algunos reaserchers. El criticismo más persistente es tht de hecho hay un mejoramiento en algún índice de bienestar de los pobres people y en algunos casos una disminución de las desigualdades, pero no como el resultado de las políticas económicas eficientes, pero el groth de expenditures social. El siguiente gráfico, elaborado por el economista Nora Lusting muestra el crecimiento del PIB la primera década del siglo. Los datos correspondientes a los años más recientes deben ser actualizados, pero indican la profundización de esta tendencia. Como muestra la tabla, los gobiernos de izquierda tuvieron mejores resultados económicos que la política ortodoxa orientada. La explicación no es sólo económica; por contrario, las políticas macroeconómicas aparecen como la variable dependiente de la política, en el sentido de que "la política" significa la política de inclusión sociales. En contra de la dictadura, donde la exclusión de la política y la rendición de cuentas son la regla, en un entorno democrático, la participación y la rendición de cuentas funciona como una fuerza positiva para la economía. 
Las diferentes políticas económicas entre los países de América Latina tienden a una diferenciación. Hasta finales del siglo 20 era común para incluir todas las políticas de ajuste como neoliberal (Cohen y Centeno, 2006). Sin embargo, a principios del siglo 21, algunas diferencias intrarregionales impotentes son importantes. Básicamente clasificación simple puede incluir tres tipos de orientaciones (excluyendo Cuba). Ortodoxa, anti-inflacionario, las políticas macroeconómicas de estabilización (México, Colombia, Chile), las políticas macroeconómicas no ortodoxas, la promoción del mercado interior y la diversificación externa (Brasil, Argentina). Y las políticas populistas basadas en orientaciones económicas primaria exportadoras (Bolivia, Venezuela, Ecuador). El primero de los tipos puede considerarse de economías más desarrolladas, pero con muy diferentes prioridades políticas. El crecimiento sostenido, es decir, promediado por al menos una década) de la economía se puede interpretar como una función de un mejor rendimiento la política (sistema competitivo mejor partido, mejores procedimientos electorales, y en menor coste de la acción colectiva. Este círculo virtuoso -pero perdurable- se ve obligado a un compromiso gubernamental importante en el bienestar social. El tamaño de los presupuestos es un indicador de esta afirmación. Lo mismo con cápita gastos de bienestar social. Brasil, Argentina, Venezuela, Uruguay cápita, liderando la lista de mayor compromiso con el bienestar social (con excepción de Cuba), seguido por México, Chile y Colombia.

\section{La sostenibilidad de las políticas "progresistas"}

Hay un acuerdo generalizado de que los gastos de bienestar difícilmente pueden ser única base para una política distributiva. Las políticas sociales son complementarias p de las políticas económicas, sociales y políticas integrale. Desde ese punto de vista, las políticas de bienestar gubernamentales difieren en toda la región. En términos generales, las políticas de bienestar pueden analizarse desde dos perspectivas. Desde el lado de la oferta, que dependen de la continuidad y la magnitud de la asignación de recursos de los gobiernos, y de su 
focalización en la población pobre. La mayoría de los gobiernos utiliza los gastos como un mecanismo clientelar. Este mecanismo se ve afectado por la competitividad electoral. Prima fascie, es razonable concluir que la existencia de una oposición de izquierda competitiva sea indicativa de la apertura del sistema político. La competencia electoral bajo reglas justas puede ser un mecanismo muy eficaz de rendición de cuentas y el control del mecanismo de clientelismo distribucional. La investigación futura debe comparar el mecanismo de clientelismo entre diferentes democracias políticas de América Latina, pero la tesis principal es que el factor de la izquierda en un indicador de la consolidación democrática. En el mismo sentido, la demanda de bienes públicos por la ciudadanía debe abordarse teniendo en cuenta la "recepción" de los subsidios y fondos en la población. Una forma interesante debe ser la introducción de las variables sugeridas por el fallecido Charles Tilly (2007). Costos más bajos de participación normalmente significan menores a la coacción. Normas legales y fuertes compromisos con la legalidad significan $d$ una mayor intolerancia a la impunidad de los ataques a los derechos humanos y la corrupción ogubernamental y corporativa. El bienestar humano es la prueba de progreso democrático, y viceversa.

No es sorpresivo que los llamados nuevos gobiernos de izquierda tienen los mayores compromisos con los gastos sociales. Pero otra cosa es su constituyente y continuidad, y el significado en la formación de la ciudadanía, sería precipitado hacer una valoración definitiva, ya que es necesario tomar en cuenta en el mayor lapso de tiempo de la continuidad. En los últimos años la financiación de los problemas sociales ha sido inestable y precaria, sobre todo porque están vinculados a los recursos de las actividades económicas externas, y a los ingresos por exportaciones de bienes primarios. Los presupuestos sociales serán mejores si la base fiscal de la capacidad del gobierno para introducir políticas progresistas fiscales se sostiene menos en el consumo y más en las ganancias de la actividad mercantil. Otra diferencia es si la financiación de los presupuestos sociales se asocian al sector extractivo ("ordeñar la vaca") o gravar el consumo y los ingresos de los particulares y las empresas. En Amé- 
rica Latina esta base es pequeña en comparación con los países de la OCDE.Es realista para avanzar en la conjetura de que la sostenibilidad a largo plazo de los programas de bienestar dependerá de la sostenibilidad de una base de impuestos ampliada (OEA-PNUD, 2010).

El tercer aspecto tiene que ver con el impacto de la financiación de la asistencia social a la población objetivo. La característica de la política de bienestar en relación con las redes institucionales políticos. Sin embargo, la dependencia política del programa social es más intensa en países como el llamado populistas, y en países con un sistema social de rendición de cuentas, al igual que México. En estos países el clientelismo parece estar más arraigada en la política como de costumbre políticas- La política del clientelismo debe ser también afectada por la debilidad de la rendición de cuentas regionales y locales. Por ejemplo, en Brasil, la centralización de los programas sociales no sólo beneficia al poder presidencial, pero también mejora la eficacia de los programas. En México el creciente papel de los gobernadores locales, exentos de la cadena de rendición de cuentas provoca un uso más extremo de los recursos públicos con fines electorales, además de instantáneas fortunas personales. La responsabilidad pública es una característica de un sistema político eficaz y moderno. En los sistemas democráticos es vital. Los déficits de rendición de cuentas pueden ser el talón de Aquiles de las políticas sociales.

El clientelismo es un mecanismo para comprar la lealtad social para los votos. Como era de esperar, el clientelismo se ve afectada por la competencia del partido y de los mecanismos de rendición de cuentas. En un sistema de partidos competitivo, menor es captura política de los recursos públicos. Una segunda proposición es que el clientelismo es un importante componente de la reactivación del presidencialismo en toda la región. Este hecho puede indicar la debilidad del entramado democrático. Si los partidos y el legislativo están inmersos en la captura de los recursos públicos, la concentración de poder en el ejecutivo puede ser una tentación difícil de resistir, y incluso justificarla en nombre de la gobernabilidad. Paralelamente, la participación de la población como agentes activos en el diseño de las políticas puede introducir una configuración real de "interfaces" 
de un círculo virtuoso de bienestar y la política distributiva. (Solimano, 2005; Treversa-Tejero, 2008; CEPAL, 2010; Guerrero, 2006; Huber, 2008; Potencia, 2009; Norges, 2010; OEA.PNUD, 2010).

El aparente éxito de los gobiernos "izquierdistas" de América del Sur es notable. Pero dependerá de la consolidación de la oferta de recursos. La capacidad de construir una coalición política capaz de promover $n$ base tributaria más progresiva (OEA-PNUD, 2010), y la "democratización" de las políticas de bienestar clientelistas es el mayor desafío de todos los países de América Latina. Por cierto, las políticas de populismo en función de los recursos de los bienes primarios y las políticas de subsidencia son una alternativa auto-limitada. El principal punto de interés para los científicos políticos es que ambos lados del juego, la consolidación de suministro de recursos (política fiscal) y las políticas sociales, son muy dependientes de las variables políticas e institucionales. Si status quo es una posición de auto-derrota, el cambio que requiere que las instituciones políticas proporcionan un canal de bajo costo para el surgimiento de coaliciones desafiantes que canalicen la acción colectiva para la gente común. En el sentido de que una menor dependencia de los proveedores de asistencia social del Estado y menos miedo a la represión para participar en la oposición y en las comunidades de auto-organización.

\section{CODA III. ¿Puede una anomalía cambiar un paradigma?}

La respuesta común es un no cauteloso. Przeworski y sus colaboradores $(1993 ; 2000)$ compararon el desempeño económico, incluyendo la capacidad de distribución de los diferentes sistemas políticos y concluir con pesimismo. La democracia debe ser defendida y preservada por sus libertades personales y sociales, pero su eficiencia distributiva no está garantida. Diversos analistas han puesto a prueba los conocimientos Przeworski y Asociados, con rechazos parciales. Carles Boix y Susn Stokes, que se centra en un proceso de modernización, llegaron a la conclusión de que la democracia puede producir algunos puestos políticos en la forma de atenuar las desigualdades del proceso de modernización anterior, Acemoglu y Richardon (2003) 
han hecho preguntas similares y concluye que las perspectivas no son muy optimista, no sólo por las élites pueden bloquear el surgimiento de nuevas coaliciones distributivas, sino porque el ámbito financiero mundial está en contra de las políticas distributivas.

La inconclusión de la investigación real produce un estado precario paradójico. La democracia no puede resolver las desigualdades, pero producirlo y subsiste dentro. Esta conclusión está en contra de algunas premisas básicas de la teoría democrática moderna. La respuesta común es un no cauteloso. Przeworski y sus colaboradores (1993; 20000) compararon el desempeño económico, incluyendo la capacidad de distribución de los diferentes sistemas políticos y concluir en un pesimista. La democracia debe ser defendida y preservada por sus libertades liberales, pero no su eficiencia distributiva. Diversos analistas han puesto a prueba los conocimientos Przeworski y Asociados, con rechazos parciales. Boix y Stokes (2003) que se centra en un proceso de modernización, llegaron a la conclusión de que la democracia puede producir algunos puestos políticos en la forma de atenuar las desigualdades del proceso de modernización anterior, Acemoglu y Richardon (2003) han hecho preguntas similares y concluye que las perspectivas no son muy optimista, no sólo por las élites pueden bloquear el surgimiento de nuevas coaliciones distributivas, sino porque el ámbito financiero mundial está en contra de las políticas distributivas.

La inconclusión de la investigación real produce un estado precario paradójico. La democracia no puede resolver las desigualdades, pero los procesos democráticos pueden agravarlas. Esta conclusión está en contra de algunas premisas básicas de la teoría democrática moderna. Por ejemplo, la tesis de Robert Dahl fue que la democracia se erige en el imperativo categórico de la igualdad política. La igualdad política ha sido la piedra fundacional de la construcción democrática de autores tan diferenciados como Rousseau y Tocqueville. Pero el trabajo de Dahl fue en los enlaces íntimos entre la igualdad política y la distribución social y económica de los bienes materiales. La democracia está en las libertades liberales, como la igualdad política, pero la igualdad política no es una cualidad etérea, sino una expresión 
de las capacidades de los ciudadanos para actuar democráticamente. Como ciudadanos, el derecho al voto de los derechos de acceso a la capacidad política de la democracia puede funcionar. Los correlatos sociales y económicos de la igualdad política están en el centro de la teoría democrática. Sin embargo, los que impregnan las desigualdades sociales y económicas en menoscabo de las políticas democráticas. Por lo general, las desigualdades económicas están asociadas a las asimetrías de poder político, y las asimetrías políticas refuerzan las desigualdades económicas. Este ciclo perverso es contrario al espíritu de la democracia, porque la democracia está relacionada con la igualdad política entre todos los ciudadanos adultos. Un informe reciente de APSA sucintamente señala que "las desigualdad económicas dentro de los países en vías de desarrollo permites a las élites de establecer políticas e instituciones que producen patrones de desarrollo que favorecen desproporcionadamente a su propio interés. Desigualdades nacionales también les permite resistir los cambios institucionales (potencialmente) útiles “(APSA, 2005, p.1)

¿Es América Latina un caso especial para probar la tesis de que la desigualdad no representa una amenaza grave para la democracia? En la fase de transición, la democratización sigue sin resistencia política y las nuevas élites no Impulse políticas distributivas. Por contrario, el enfoque de la economía estaba en contra de las políticas distributivas. El resultado fue un aumento de las desigualdades. La distancia entre los que tienen y no han aumentado drásticamente. En el escenario base de la economía mundial, el crecimiento de las personas marginadas aumentará rápidamente en los próximos años. Como la igualdad política no es una cáscara vacía ni una invocación mítica, debe ser apoyada por alguna distribución básicamente justa de los bienes y oportunidades. La medida de la cuántica de que es suficientemente "justo" en un pacto social puede variar a través de las sociedades, pero hay criterios comunes. Desigualdades persistentes son sistémicas y simplemente no son buenas para el funcionamiento democrático. Los regímenes democráticos pueden preservarse induciendo más igualitarismo social. La ciudadanía es un sustantivo sólo cuando se llena de una disminución de las desigualdades 
"duraderas". La cuestión distributiva no implica una relación fija de distribución de ingresos y otros bienes materiales, sino una tendencia donde las desigualdades sociales se rechazan efectivas de generación en generación. La misma percepción social de la situación distributiva depende, entonces, de las expectativas sobre el futuro de distribución. Cuando estas expectativas son más bajas, la legitimidad de los canales regulares de la movilización social podría estar en condiciones precarias. La apertura de canales ilegales a las actividades económicas es la última oportunidad para la movilidad social. No sólo para los mercados ilegales u oscuros, buto para actividades ilegales y criminales. (OEA.PNUD, 2010). Pero también, cuando se perciben que los canales políticos son precarios, ilegítimos o parciales, el riesgo de una grieta profunda, o más a menudo, la ineficiencia institucional y la incapacidad para responder a los desafíos internos y externos es real y autodestructiva. El régimen está deteriorando el Estado sufre un proceso de deterioro. Democracias deterioradas produce estados débiles.

\section{REFERENCIAS}

ALCÁNTARA SÀEZ, M.; FREIDENBERG, F. Los partidos políticos en América Latina. In: Manual de gerencia política, Instituto Nacional Demócrata, 2001.

ALTMANN, D. \& CASTTIGLIONI, R. Democratic Quality and Human Development in Latin America: 1972-2001. CAPS, Canadian Kournalm of Political Science, Vol. 42, nº.2, June, 2009.

APSA. The Persistent Problem. Inequality, Difference, and the Challenge of Development. American Political Science Association, Report of the Task Force on Difference, Inequality, and Developing Societies, July, 2008.

BANCO MUNDIAL. Gobernabilidad Democrática en México. Más allá de la captura del Estado, México. Banco Inter-Americano de Reconstrucción y Fomento, Informe no. 37293-MX, 2007.

BERMEO, N. Does Electoral Democracy Boost Economic Equality? 
Journal of Democracy, Vol. 20, n. 4, October, 2009.

BETHELL, L. (Coord.). Historia de America Latina, Vol. XII, Cambridge University Press, 2011.

BOIX, C. Democracy and Redistribution. Cambridge University Press, 2003.

BOIX, C. y STOKES, S. C. Endogenous Democratization. World Politics, Vol. 55, Julio, pp.517-549, 2003.

BORGES, A. Já não fazem máquinas políticas como antigamente: competição e mudança nos estados brasileiros. Curitiba, Revista de Sociologia Política, Vol.18, no.35, 2010.

BORSANI, H. Gobiernos de izquierda, sistema de partidos y los desafíos para la consolidación democrática en América Latina. Stockholm Review of Latin American Studies, Issue no.3, December, 2008.

CHONG, A. Inequality, Democracy, and Persistence: Is There a Political Kuznets Curve? Inter-American Development Bank and Georgetown University, Working Paper 445, April, 2001.

CHONG, A.; LUCERO, M. On Compulsory Voting and Income Inequality in Cross Section of Countries. Inter American Development Bank, Working Paper 533, May, 2005.

COHEN, J. N.; CENTENO, M. Á. Neoliberalism and Patterns of Economic Performance 1980-2000. ANNALS, AAPSS, 606, July, 2006.

CÓRDOVA, A.; SELIGSON, M. A. Economic Crisis and Democracy in Latin America, APSA, PS, October, 2008.

CORREA, M. Partidos políticos y representación en América Latina. Perspectivas desde el barómetro de las Américas (LAPOP), nº. 36, 2010.

DAHL, R. Poliarchy. Yale University Press, 1971.

DE FERRANTI, D., et.al. Inequality in Latin America and the Caribbean. Breaking with History? World Bank Latin American and Caribbean Studies, Advanced Conference Edition, 2003.

DIAMOND, L. Thinking About Hybrid Regimes. Journal of Democracy, Vol.13, no.2, April, 2002.

ECHEVERRI-GENT, J. New approaches to the Political of Inequality in Developing Countries, PS, October, 2009. 
GUERRERO I.; WALTON, M. Las trampas de la desigualdad y su vínculo con el bajo crecimiento de México. Banco Mundial, 2006.

HOPKIN, J. The politics of Piketty: what political science can learn from, and contribute to, the debate on Capital in the Twenty-First Century. The British journal of sociology, Vol.65, n4, pp.678-695, 2014.

HUBER, E.; PRIBBLE, J.; NIELSEN, F.; STEPHENS, J. Politics and Inequality in Latin America and the Caribbean. American Sociological Review, Vol. 71, December, 2006.

HUBER, E.; MUSTILlO, T. y STEPHENS, J. Politics and Social Spending in Latin America. The Journal of Politics, Vol. 70, nº. 2, Abril, 2008.

KAUFFMAN, R. R. The Political Effects of Inequality in Latin America. Some Inconvenient facts. Comparative Politics, n'.41, April, 2009.

LAGOS, M. Latin America, Diversity Crisis, National Endowment for Democracy, Journal of Democracy, Vol.19, nº.1, pp.112-116, 2008.

LATINO BAROMETRO. Informe 2010, Septiembre, Santiago de Chile.

LIPSET, S. M. Some Social Requisites of Democracy: Economic Development and Political Legitimacy. American Political Science Association. American Political Science Review, Vol. 53, nº. 1, pp. 69-105, 1959.

LÓPEZ, S. Partidos desafiantes en América Latina.: representación política y estrategias de competencia de las nuevas oposiciones. Revista de ciencia politica, Santiago, Vol. 25, nº.2, 2005.

LUSTING, N. Hacia un marcos para entender la política de reducción de la pobreza y la desigualdad. Desigualdad y pobreza y la "nuevas izquierda" de América Latina. Woodrow Wilson Center-FLACSO. Latin American Program Report, nº. 6, October, 2009.

MANN, M. The Sources of Social Power, The Rises of Classes and national States, 1790-1914, Vol. II, Cambridge: Cambridge University Press, 1993.

MILANOVIC, B. Globalization and Inequality, The Ralph Miliband Lectures on Inequality. London School of Economics and Political Science, 2005.

MUNCK, G.; VEKUILEN, J. Conceptualizing and Measuring Democracy. Evaluating Alternative Indices. Comparative political studies, Vol.35, no.1, pp.5-34, 2002.

MUNCK, G. L.; SNYDER, R. Passion, Craft, and Method in Comparative 
Politics. Baltimore, MD: The Johns Hopkins University Press, 2007.

NAVIA, P. Partidos políticos como antídoto contra el populismo en América Latina. Pontificia Universidad Católica de Chile, Revista de Ciencia Política, Vol. XXIII, n.1, 2003.

OEA-PNUD. Nuestra democracia. OEA, PNUD, FCE, México, 2010.

PIKETTY, T. Capital in the 21st Century. Cambridge: Harvard University Press, 2014.

POWER, T. J. Compulsory for Whom? Mandatory Voting and Electoral Participation in Brazil, 1986-2006. Journal of Politics in Latin America, Vol.1, n.1, pp.97-122, 2009.

PROGRAMA DE LAS NACIONES UNIDAS PARA EL DESARROLLO. La Democracia en America Latina - Hacia una democracia de ciudadanas y ciudadanos, Ed. Aguilar, ASltea, Taurus Alfaguara, 2004.

PRZEWORSKY, A.; LIMONGI, F. G. Political Regimes and Economic Growth. Journal of Economic Perspectives, Vol. 7, $\mathrm{n}^{\mathrm{o}} .3$, pp.51-69, 1993.

PRZEWORSKI, A. Una defensa de la concepción minimalista de la democracia. Revista Mexicana de Sociología, Vol.59, nº.3, julioseptiembre, 1997.

PRZEWORSKI, A.; ÁlVAREZ, M.; CHEIBAUB, J. A. y LIMONGI, F. Democracy and Development. Political institutions and well-Being in the world 1950-1990, New York, Cambridge University Press, 2000.

SELIGSON, M. A. The Rise of Populism and the Left in Latin America. Journal of Democracy. Vol. 18, nº 3, July, 2007.

SEN, A. Development as Freedom. New York: Anchor Book, 1999.

SOLIMANO, A. The middle class and the development process. ECLA, 2008.

STEVEN, D.; BISHIN, B. G. and BARR, R. R. Authoritarian Attitudes, Democracy and Policy Preferences among Latin American Elites. Midwest Political Science Association, American Journal of Political Science. Vol. 50, nº. 3, pp. 606-620, 2006.

ECHEVARRI-GENT, J. (Ed.). Symposium. New Appoaches to the Politics of Inequality in Developing Countries, APSA, PS, October. 
TILLY, C. Durable Inequality. Berkeley. Los Angeles. University of California Press, 1998, 2004. . Democracy. Cambridge University Press, 2007.

TRAVERSA-TEJERO, F. Democracia y redistribución en América Latina. Stockholm Review of Latin American Studies. Issue no. 3, December, 2008.

WALTON, M; BEBBINGTON, A. J.; DANI, A. A.; HAAN, A. de (Eds.). Institutional paths to Equity, Addressing Inequality Traps. Washington D.C., World Bank, 2007.

ZAVATTO, D. G. Participación electoral en América Latina. Tendencias y perspectivas 1978-2000. Elecciones, Vol. 2, n. 23, 2006.

UNDP, Human Development Report. Sustainability and Equity: A Better Future for All. Sustainability and Equity: A Better Future for All. UNDP-HDRO Human Development Reports, 2011.

Recebido: 16/06/2015. Aprovado: 20/10/2015. 